\title{
Séquestration osseuse sur l'extrémité distale de la ligne mylo-hyoïdienne : à propos de huit cas
}

\author{
Bone sequestration on the distal end of the mylohyoid line : \\ a report of eight cases
}

IMEN BOUZOUITA', SIMON ABI NAJM'1', TOMMASO LOMBARDI², JACKY SAMSON

\section{RÉSUMÉ}

II s'agit d'une présentation de huit cas de séquestre osseux développé sur l'extrémité distale de la ligne mylohyoïdienne, examinés dans la Division de Stomatologie, Chirurgie orale et Radiologie dento-maxillo-faciale de l'Ecole de Médecine dentaire de l'Université de Genève.

L'extrémité distale de la ligne mylo-hyoïdienne comporte un relief osseux constitué d'os compact, peu vascularisé, qui peut parfois se nécroser spontanément.

La présentation de huit cas permet de préciser les signes cliniques, radiologiques et histologiques de cette affection dont l'étiopathogénie n'est pas totalement élucidée. II semble toutefois qu'il s'agit d'une nécrose osseuse ischémique, secondaire à un épisode inflammatoire. (Med Buccale Chir Buccale 2009 ; 15 : 49-57).

mots clés: ligne mylo-hyoïdienne, séquestre osseux, ischémie, bisphosphonates

médecine buccale chirurgie buccale

VOL. $15, \mathrm{~N}^{\circ} 1$ 2009

page 49

\section{SUMMARY}

Eight cases of bone sequestration on the distal end of the mylohyoid line, examined in the Division of Stomatology, Oral Surgery and Radiology of the Dental School of the University of Geneva are presented.

The relief of the distal end of the mylohyoid line is made by a compact bone, with low blood supply. A necrosis of this small area of bone may occur spontaneously.

In this article, we present the clinical symptoms, the radiological images, and the histhopathological aspects of this entity, in which the etiology remains still to be clarified. It seems like there is an inflammatory process followed by an ischemic bone necrosis. (Med Buccale Chir Buccale 2009 ; 15: 49-57).

key words: mylohyoid line, bone sequestration, ischemia, bisphosphonates 
médecine

buccale

chirurgie

buccale

VOL. $15, \mathrm{~N}^{\circ} 1$ 2009

page 50
Dans les maxillaires, un séquestre osseux peut se développer après une extraction dentaire, une intervention de chirurgie parodontale, une infection, une fracture de l'os alvéolaire ou une fracture comminutive, une ostéonécrose (ostéoradionécrose, ostéonécrose due aux bisphosphonates). En l'absence de traitement, le séquestre est éliminé spontanément par voie transépithéliale dans un délai très variable ${ }^{[7,9]}$.

Exceptionnellement, un séquestre osseux peut se développer sur l'extrémité distale de la ligne mylo-hyoïdienne, en dehors de tout contexte pathologique. La ligne mylo-hyoïdienne forme une crête oblique de bas en haut et d'avant en arrière, barrant la face interne de la branche horizontale de la mandibule (Fig. 1). Dans son tiers postérieur, le relief osseux est plus marqué. Son extrémité distale qui se trouve à hauteur de la dent de sagesse et à 7-8 $\mathrm{mm}$ du sommet de la crête alvéolaire dentée, est constituée par un os très compact et peu vascularisé (Fig. 2).

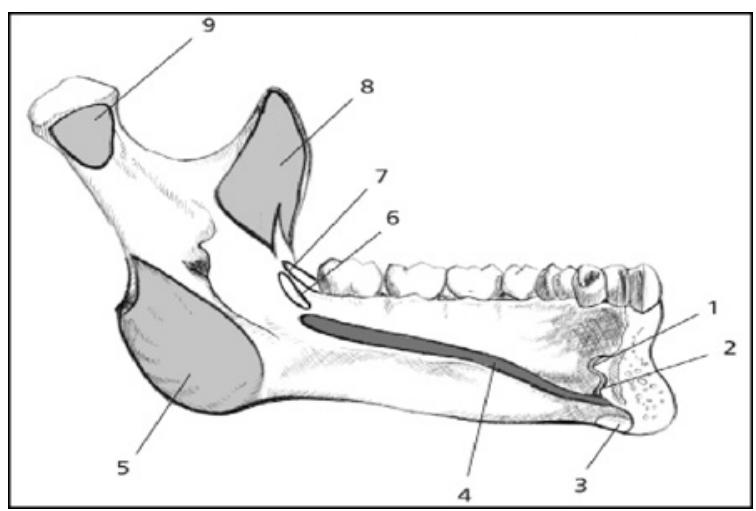

Figure 1: Insertion des muscles sur la face interne de la mandibule. La ligne mylo-hyoïdienne correspond à l'insertion du muscle mylo-hyoïdien [4]. (1. génio-glosse, 2. génio-hyoïdien, 3. digastrique, 4. mylo-hyoïdien, 5. ptérygoïdien médial, 6 . constricteur supérieur du pharynx, 7. buccinateur, 8. temporal, 9. ptérygoïdien latéral).

Insertion of the muscles on the lingual surface of the mandible. The mylohyoid line represents the attachment area of the mylohyoid muscle. (1. genio-gloss, 2. genio hyoid, 3. digastric, 4. mylohyoid, 5. medial pterygoid, 6. superior pharyngeal constrictor, 7.buccinator, 8. temporalis, 9. lateral pterygoid).

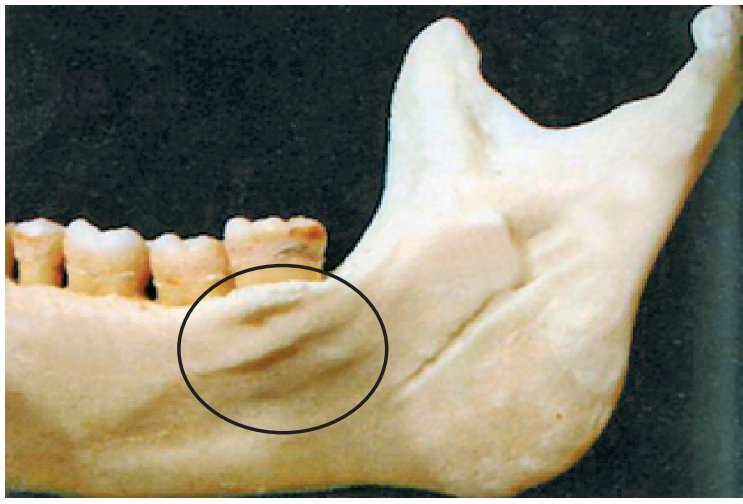

Figure 2: Importance du relief de l'extrémité distale de la ligne mylo-hyoïdienne [3].

Importance of the ridge of the distal end of the mylohyoid line.

\section{MATERIEL ET METHODE}

Cette étude concerne 9 cas de séquestres osseux, intéressant la région de l'extrémité distale de la ligne mylo-hyoïdienne, observés dans la Division de Stomatologie, Chirurgie orale et Radiologie dento-maxillo-faciale de l'Ecole de Médecine dentaire de l'Université de Genève.

Les principales données concernant ces cas (âge, sexe, localisation anatomique, symptomatologie, évolution et traitement effectué) sont présentées dans le Tab. 1. Un examen radiographique et un examen histopathologique n'ont pas été effectués systématiquement pour chaque cas.

\section{RESULTATS}

Le développement de ce séquestre a été observé chez neuf adultes ( 3 femmes, 6 hommes) ayant un âge moyen de 54,9 ans. L'anamnèse n'a pas apporté d'éléments contributifs, excepté pour le dernier patient qui était traité par bisphosphonates pour un myélome multiple. On n'a retrouvé aucune notion de traumatisme ou de traitement dentaire récents ; les dents adjacentes au séquestre étaient présentes, vitales et sans atteinte parodontale pouvant être à l'origine d'une ostéomyélite.

Le séquestre s'est développé sur l'extrémité distale de la ligne mylo-hyoïdienne, excepté pour le patient traité par bisphosphonates où la lésion ne semblait pas située exactement sur l'extrémité 
Tableau 1 : Données concernant les neufs cas présentés.

Data relative to the nine cases reported.

( $H$ : homme, $F$ : femme, AB : antibiothérapie, AINS : anti-inflammatoires non stéroïdiens, OPT : orthopantomogramme).

\begin{tabular}{|c|c|c|c|c|c|c|c|}
\hline cas & $\begin{array}{l}\text { sexe } \\
\text { et } \\
\text { âge }\end{array}$ & anamnèse & localisation & symptômes & radiographie & traitement & $\begin{array}{c}\text { examen } \\
\text { histopathologique }\end{array}$ \\
\hline$n^{\circ} 1$ & H52 & - & $\begin{array}{c}38 \\
\text { linguale }\end{array}$ & douleurs & - & séquestrectomie & séquestre osseux \\
\hline$n^{\circ} 2$ & F53 & - & $\begin{array}{c}38 \\
\text { linguale }\end{array}$ & $\begin{array}{l}\text { douleurs } \\
\text { ulcération }\end{array}$ & $\begin{array}{l}\text { coupe frontale } \\
\left(\text { Scanora }^{\circledR}\right)\end{array}$ & $\begin{array}{c}\text { séquestrectomie, } \\
A B\end{array}$ & séquestre osseux \\
\hline$n^{\circ} 3$ & F61 & - & $\begin{array}{c}38 \\
\text { linguale }\end{array}$ & $\begin{array}{l}\text { douleurs } \\
\text { irradiantes }\end{array}$ & OPT & $\begin{array}{c}\text { ostéo-ectomie } \\
\text { corticoïdes, AB } \\
\text { Tissucol }^{\circledR}\end{array}$ & - \\
\hline$n^{\circ} 4$ & H61 & - & $\begin{array}{c}48 \\
\text { linguale }\end{array}$ & $\begin{array}{l}\text { douleurs } \\
\text { œdème }\end{array}$ & apicale & AB, AINS, corticoïdes & - \\
\hline$n^{\circ} 5$ & $\mathrm{H} 45$ & - & $\begin{array}{c}48 \\
\text { linguale }\end{array}$ & $\begin{array}{l}\text { douleurs } \\
\text { exposition }\end{array}$ & apicale & $\begin{array}{c}\text { ostéo-ectomie, AINS, } \\
A B\end{array}$ & - \\
\hline$n^{\circ} 6$ & H54 & - & $\begin{array}{c}48 \\
\text { linguale }\end{array}$ & $\begin{array}{l}\text { œdème } \\
\text { exposition }\end{array}$ & - & $\begin{array}{c}\text { ostéo-ectomie, AINS, } \\
A B\end{array}$ & séquestre osseux \\
\hline$n^{\circ} 7$ & F46 & - & $\begin{array}{c}37 \\
\text { linguale }\end{array}$ & douleurs & apicale & $\begin{array}{c}\text { ostéo-ectomie, AINS, } \\
A B\end{array}$ & séquestre osseux \\
\hline$n^{\circ} 8$ & H67 & - & $\begin{array}{c}38 \\
\text { linguale }\end{array}$ & douleurs & - & séquestrectomie & séquestre osseux \\
\hline$n^{\circ} 9$ & $\mathrm{H} 75$ & $\begin{array}{l}\text { myélome } \\
\text { multiple }\end{array}$ & $\begin{array}{c}37 \\
\text { linguale }\end{array}$ & $\begin{array}{l}\text { exposition } \\
\text { indolore }\end{array}$ & OPT & $\begin{array}{c}\text { ostéo-ectomie, AB } \\
\text { Tissucol }{ }^{\circledR}\end{array}$ & séquestre osseux \\
\hline
\end{tabular}

médecine buccale chirurgie buccale

VOL. $15, \mathrm{~N}^{\circ} 1$ 2009

page 51 distale de la ligne mylo-hyoïdienne, mais un peu plus en avant et plus haut.

Lors de la première consultation, il existait dans la région de l'extrémité distale de la ligne mylohyoïdienne :

- des douleurs vives et irradiantes (7 cas sur 9),

- un érythème accompagné d'un œdème (2 cas sur 9),

- une exposition osseuse (3 cas sur 9),

- une ulcération sans exposition osseuse (1 cas sur 9).

Dans les 3 cas où il y avait une exposition osseuse, le séquestre était en voie de formation : en surface, l'os nécrosé était séparé en partie de l'os sain et on pouvait en suivre les limites avec une sonde. Dans les autres cas où les limites du séquestre n'étaient pas apparentes, une ostéo-ectomie a été effectuée, associée à une prescription d'antibiotiques et d'AINS. La cicatrisation muqueuse s'est effectuée en 1 à 2 semaines dans tous les cas. Chez le patient traité par bisphosphonates, l'exposition osseuse était indolore ; une nouvelle exposition est apparue 31 mois plus tard, dans la même région mais dans une position plus crestale.

Les radiographies périapicales et l'orthopantomogramme effectués ne montraient aucune modification de la structure osseuse. La seule coupe frontale réalisée (cas $\left.n^{\circ} 2\right)$ montre une opacité irrégulière dans la région concernée.

L'examen histopathologique, lorsqu'il a été effectué, a confirmé le diagnostic de séquestre osseux de la branche horizontale de la mandibule. 


\section{DISCUSSION}

Lors du développement d'un séquestre osseux sur la ligne mylo-hyoïdienne, on distingue trois phases au cours de l'évolution spontanée:

- phase inflammatoire (douleurs, érythème et œdème),

- phase d'exposition et de formation du séquestre,

- phase d'élimination du séquestre et de cicatrisation par seconde intention.

Dans un premier temps, le processus se manifeste par des douleurs souvent intenses, permanentes, lancinantes, qui peuvent irradier vers I'hémiface et l'oreille homolatérales. Puis il apparait un érythème et bientôt un œdème dans la région de l'extrémité distale de la ligne mylo-hyoïdienne ; cette phase est souvent accompagnée par une exacerbation des douleurs (Fig. 3). A ce

médecine buccale chirurgie buccale

VOL. $15, \mathrm{~N}^{\circ} 1$ 2009

page 52

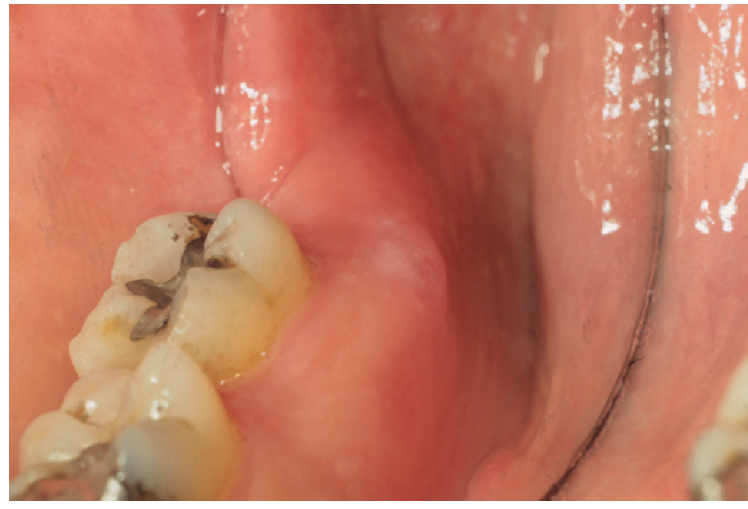

Figure 3 : Muqueuse érythémateuse et œdématiée, avec de vives douleurs (cas n'4).

Acute pain and mucosa with œdema and eythema. (case $n^{\circ} 4$.)

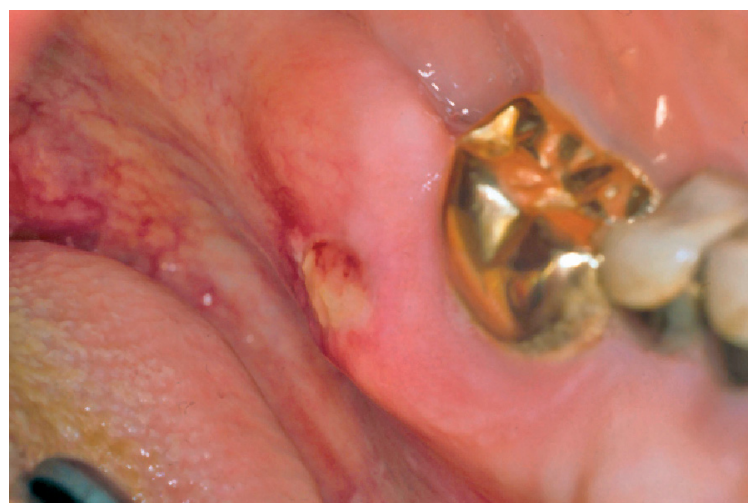

Figure 4 : Ulcération nécrotique avant le stade d'exposition osseuse (cas $n^{\circ} 3$ ).

Necrotizing ulceration before bone exposure (case $n$ ํ).

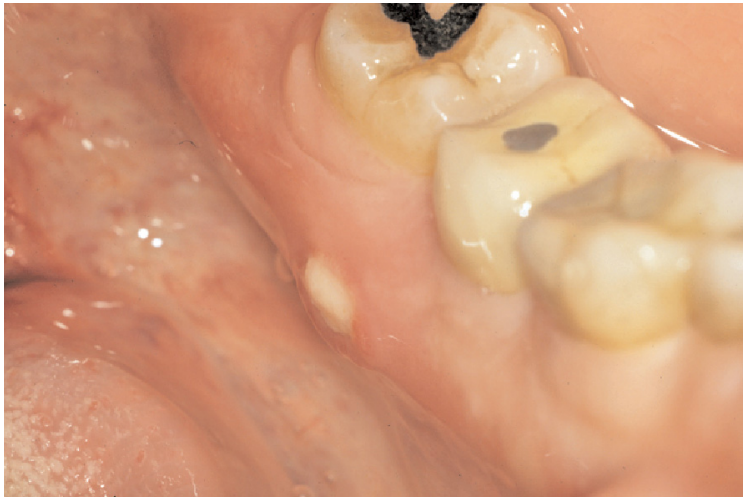

Figure 5 : Exposition osseuse : séquestre en voie de formation (cas $\left.n^{\circ} 1\right)$.

Bone exposure : ongoing bone sequestration (case $n^{\circ} 1$ ).

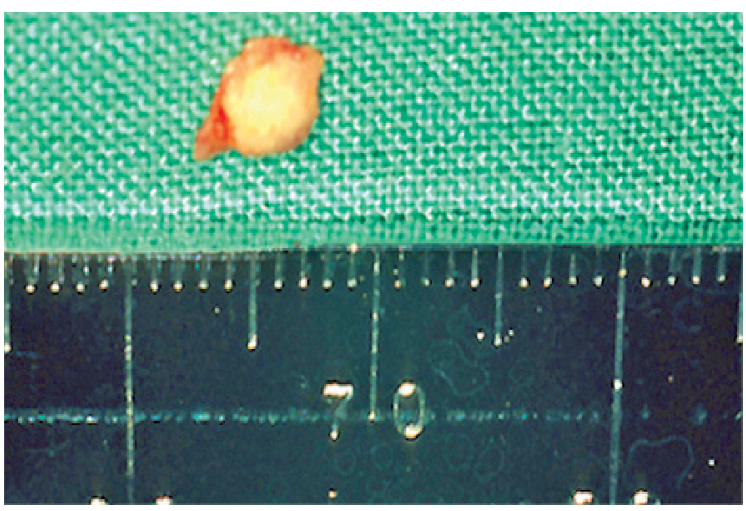

Figure 6 : Séquestre éliminé (cas $\left.n^{\circ} 1\right)$.

Exfoliation of the bone sequestrum (case $n^{\circ} 1$ ).

stade, le diagnostic est rarement évoqué. Cinq à sept jours après l'apparition des douleurs, une ulcération arrondie, de 4 à $5 \mathrm{~mm}$ de diamètre est observée à 9-10 mm du bord libre de la gencive ou du sommet de la crête alvéolaire dentée (Fig. 4). Cette ulcération met à nu un os compact, ayant une surface un peu convexe, légèrement irrégulière, non sensible à la palpation (Fig. 5). Les tissus mous adjacents sont érythémateux, œdématiés, et le moindre contact exacerbe les douleurs. Un séquestre de 3 à $5 \mathrm{~mm}$ de diamètre se forme progressivement tandis que les douleurs régressent (Fig. 6). L'élimination du séquestre marque le début de la cicatrisation par seconde intention.

Plusieurs hypothèses étiopathogéniques ont été proposées. Elles font intervenir :

- des facteurs locaux : perte des molaires, restaurations incorrectes ne rétablissant pas la forme anatomique des dents ${ }^{[7]}$, 
- des facteurs génétiques prédisposants : torus mandibulaires proéminents [9], survenue d'aphtes ${ }^{[8]}$,

- des facteurs systémiques : diabète et déficit immunitaire [2].

Ces hypothèses sont diversement associées selon les auteurs. Le mécanisme proposé par Peeters et coll. [7], repris par la plupart des auteurs, repose sur un mécanisme étiopathogénique exogène. Il décrit la survenue d'un traumatisme favorisé par les facteurs locaux cités ci-dessus, mais aussi par l'existence d'un relief osseux sur l'extrémité distale de la ligne mylo-hyoïdienne, proéminent, tranchant et recouvert par une muqueuse libre, peu épaisse. Le processus inflammatoire entrainerait par compression une diminution de la vascularisation du périoste, donc de l'os sous-jacent. La forte densité osseuse dans cette région et la faible vascularisation de l'os associées à la diminution de la vascularisation provenant du périoste, entraîneraient une nécrose osseuse par ischémie. La nature ischémique de cette nécrose expliquerait l'intensité des douleurs. Un mécanisme endogène a également été proposé. Comme pour la nécrose aseptique de la hanche, l'ischémie pourrait être due à une altération de la paroi vasculaire, une thrombose vasculaire ou un écrasement des micro-vaisseaux. II semble exister des facteurs « ischémiants » agissant sur l'os faiblement vascularisé, ce qui provoquerait une ischémie déclenchant le processus inflammatoire, qui à son tour majorerait l'ischémie [10] ; I'ulcération se développant secondairement pour permettre l'élimination du séquestre. Le mécanisme endogène correspond à la chronologie des signes et des phases cliniques observées au cours de l'évolution. De plus, sept des patients examinés ne présentent aucun facteur génétique, local ou systémique prédisposant évident. Un seul présentait des torus mandibulaires proéminents, élément décrit comme facteur génétique prédisposant ${ }^{[9]}$.

Classiquement, le traitement associe la désinfection locale, la réalisation de bains de bouche et la prescription d'antalgiques [1]. La douleur est contrôlée par les antalgiques usuels mais il semble préférable d'y associer des antibiotiques. L'élimination spontanée du séquestre marque le début de la phase de cicatrisation. Pour accélérer l'évolution, on peut favoriser l'élimination de l'os nécrosé soit par clivage lorsque la limite du séquestre est déjà apparue, soit à la fraise dès l'apparition de l'exposition osseuse. La cavité laissée par l'élimination de l'os nécrosé peut être comblée par de la colle de fibrine. Cette façon de procéder raccourcit la phase d'état, le délai de cicatrisation restant apparemment constant en l'absence de surinfection.

Au stade initial, il semble qu'une corticothérapie systémique (prednisone 1mg/kg x 3 j) puisse stopper l'évolution vers la nécrose osseuse et la formation d'un séquestre ; la résolution des symptômes se faisant en quelques jours.

Les radiographies péri-apicales et panoramiques ne retrouvent aucune anomalie de la structure osseuse dans la région du séquestre. La radiographie occlusale ${ }^{[7]}$ et les coupes tomodensitométriques mettent parfois en évidence une image opaque, mal limitée, sur la corticale linguale de la mandibule, dans la région où le séquestre est en cours de formation.

L'examen histopathologique montre un os cortical avec des logettes ostéocytaires vides, un infiltrat inflammatoire et une disparition de la moelle osseuse (Fig. 7). Cet aspect est caractéristique de l'os nécrosé.

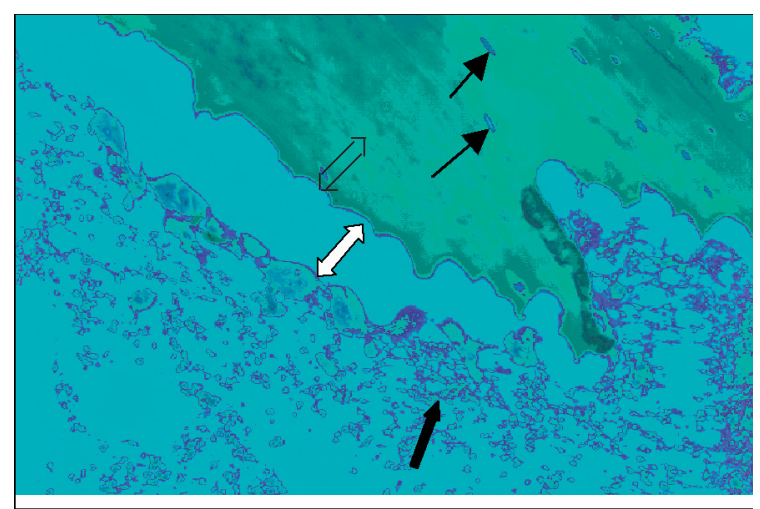

Figure 7 : Examen histopathologique d'un séquestre osseux (HES, x40) (cas $\left.n^{\circ} 1\right): \longrightarrow$ logettes ostéocytaires vides, $\Longleftrightarrow$ absence de moelle osseuse, $\rightarrow$ infiltrat inflammatoire.

Bone sequestrum caracterised by (HES, x40) (case ${ }^{\circ} 1$ ): $\longrightarrow$ empty osteocytic lacunae, $\longleftrightarrow$ absence of bone marrow, $\rightarrow$ acute inflammatory infiltrate. médecine

buccale

chirurgie

buccale

VOL. $15, \mathrm{~N}^{\circ} 1$ 2009

page 53 


\section{PRESENTATION DE TROIS CAS}

Cas $\mathbf{n}^{\circ} \mathbf{2}$ : Une patiente de 53 ans a été adressée par son chirurgien dentiste pour une douleur dans la région de la 38. A l'examen clinique, on a découvert une ulcération de la fibromuqueuse linguale, avec une exposition osseuse siégeant sur l'extrémité distale de la ligne mylo-hyoïdienne (Fig. 10). Les radiographies conventionnelles n'ont montré aucune anomalie de la structure osseuse dans la région concernée. Par contre, sur une coupe frontale réalisée avec le Scanora ${ }^{\circledR}$, on a observé une image opaque de densité légèrement inférieure à celle de l'os cortical mandibulaire, qui semblait posée sur la corticale linguale (Fig. 11).

Le séquestre osseux a été éliminé par clivage et la cicatrisation muqueuse s'est effectuée en une semaine sous antibiothérapie (amoxicilline 750 mg $x$ 3/j) (Fig. 12).

médecine buccale chirurgie buccale

VOL. $15, \mathrm{~N}^{\circ} 1$ 2009

page 54
L'examen histopathologique a confirmé le diagnostic de séquestre cortical de table interne de la mandibule.

Cas n $^{\circ} 7$ : II s'agit d'une patiente de 46 ans qui est venue consulter pour une douleur dans la région mandibulaire postérieure gauche du côté linguale. A l'examen clinique, il existait une plage érythémateuse sur la fibromuqueuse linguale à hauteur de la 37 (Fig. 8).

Les dents adjacentes étaient vitales et le sondage n'a pas mis en évidence d'atteinte parodontale. La radiographie apicale ne montrait pas d'anomalie de la structure osseuse (Fig. 9).

Des rinçages à base de chlorhexidine, des AINS (acide méfénamique 500 mg x 3/j) et une antibiothérapie (amoxicilline $750 \mathrm{mg} \times 3 / \mathrm{j}$ ) ont été prescrits pendant 5 jours, puis une ostéo-ectomie a été effectuée pour éliminer l'os exposé, l'antibiothérapie a été arrêtée au $6^{e}$ jour, devant l'évolution favorable de la cicatrisation.

La symptomatologie a régressé rapidement après l'ostéo-ectomie et la cicatrisation était terminée 15 jours après l'élimination de l'os nécrosé. L'examen histopathologique a confirmé le diagnostic de séquestre osseux.

Cas $\mathbf{n}^{\circ} 9$ : Ce patient de 75 ans a été adressé par son chirurgien dentiste pour une exposition osseuse indolore de $4 \mathrm{~mm}$ de diamètre, située à $5 \mathrm{~mm}$ du sommet de la crête alvéolaire, distale à la 36 (la 37 a été extraite il y a plusieurs années) : elle siège donc un peu au-dessus de la ligne mylo-hyoïdienne (Fig. 13). Ce patient était traité par Arédia ${ }^{\circledR}$ (pamidronate $90 \mathrm{mg} / \mathrm{mois}$ ) depuis 12 mois pour un myélome multiple. L'orthopantomogramme ne montrait aucune modification de la structure osseuse dans la région de l'exposition (Fig. 15).

Après une antibiothérapie (amoxicilline 750 mg x 3/j $x 7 \mathrm{j})$, une ostéo-ectomie à la fraise a été effectuée ; la cavité osseuse comblée avec de la colle de fibrine. L'antibiothérapie a été poursuivie pendant une semaine. La cicatrisation muqueuse s'est effectuée lentement par seconde intention. II persistait une dépression dans la zone d'exposition osseuse qui était encore visible 19 mois après l'ostéo-ectomie (Fig. 14). L'examen histopathologique de l'os exposé a confirmé le diagnostic d'une ostéomyélite nécrosante.

Le patient a été revu pour un examen de contrôle 31 mois après la première consultation : on a constaté qu'une nouvelle exposition osseuse était apparue un peu au-dessus de la précédente (Fig. 16). La radiographie apicale ne montrait aucune anomalie de la structure osseuse (Fig. 17).

Dans ce cas, les deux expositions osseuses étaient situées dans des zones voisines mais au-dessus et en avant de la zone où survient habituellement le séquestre osseux de l'extrémité distale de la ligne mylo-hyoïdienne. Ceci faisait plus suspecter une ostéonécrose due aux bisphosphonates qu'un séquestre osseux en relation avec la ligne mylohyoïdienne. De plus, dans cette dernière affection, aucun cas de récidive n'a jamais été rapporté.

D'autres cas de séquestre osseux sur la corticale linguale mandibulaire ont été décrits chez des patients traités par bisphosphonates [5,6], mais la localisation exacte des séquestres osseux n'était pas précisée par les auteurs.

\section{CONCLUSION}

La présentation de ces huit cas de séquestre osseux sur l'extrémité distale de la ligne mylohyoïdienne permet d'illustrer une affection rare et méconnue. L'os cortical formant l'extrémité distale de la ligne mylo-hyoïdienne, compact et peu vascularisé, pourrait se nécroser spontanément 


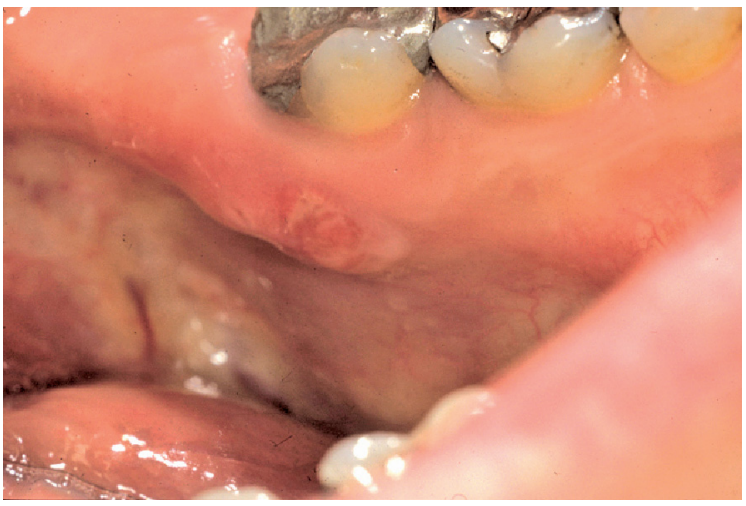

Figure 8: Ulcération de la muqueuse linguale au stade initial (cas $\left.n^{\circ} 7\right)$.

Ulceration of the lingual mucosa in the initial stage (case $\left.n^{\circ} 7\right)$.

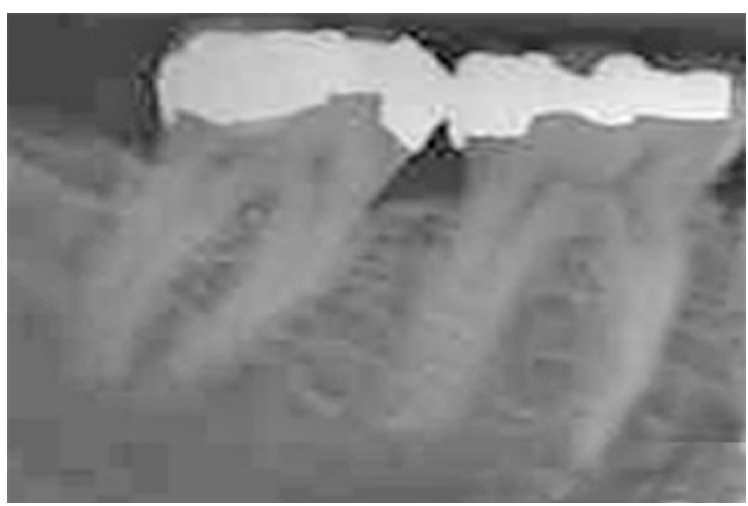

Figure 9 : Absence d'anomalie radiologique dans la région concernée (cas $n^{\circ} 7$ ).

Absence of radiological signs of pathology in the area concerned (case $n^{\circ} 7$ ).

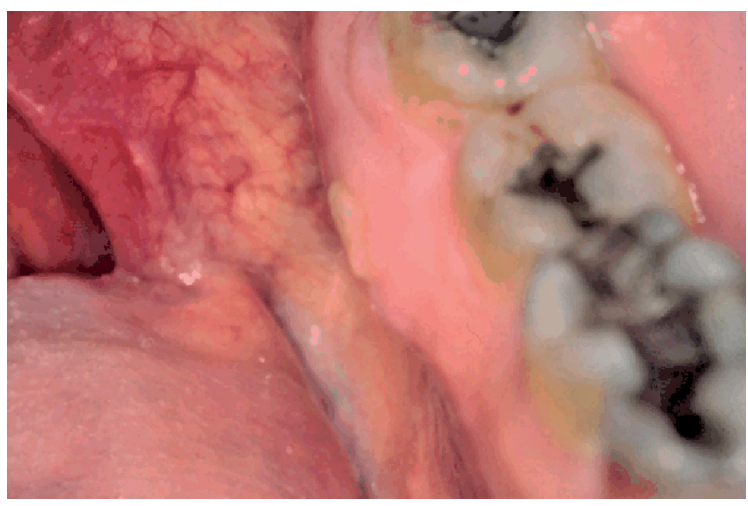

Figure 10: Ulcération et exposition osseuse sur l'extrémité distale de la ligne mylo-hyoïdienne, à hauteur de la 38 (cas $\left.n^{\circ} 2\right)$.

Ulcearation and bone exposure on the distal end of the mylohyoid line, adjacent to the lower left wisdom tooth (case $n^{\circ} 2$ ).

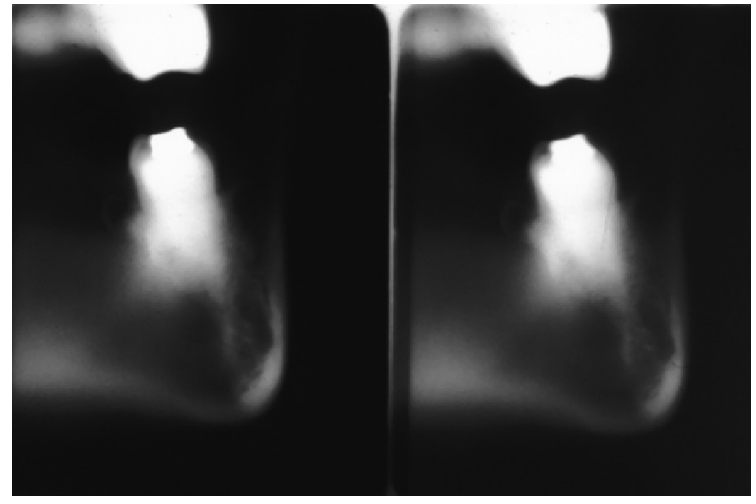

Figure 11: Discrète opacité sur la corticale linguale (Scanora ${ }^{\circledR}$, coupe frontale, cas $\left.n^{\circ} 2\right)$.

Discrete opacity on the lingual cortical bone (Scanora ${ }^{\circledR}$, frontal plane, case $\left.n^{\circ} 2\right)$.

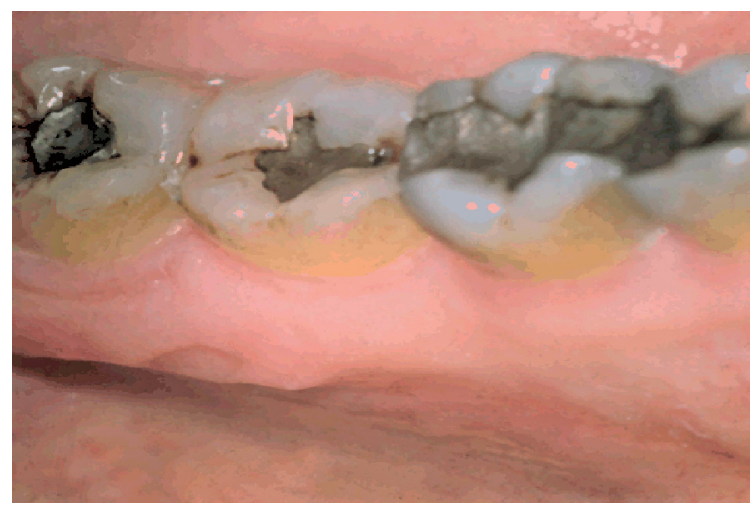

Figure 12: Cicatrisation après séquestrectomie et antibiothérapie (cas $\left.n^{\circ} 2\right)$.

Healing after sequestrectomy and antibiotherapy (case $\left.n^{\circ} 2\right)$.

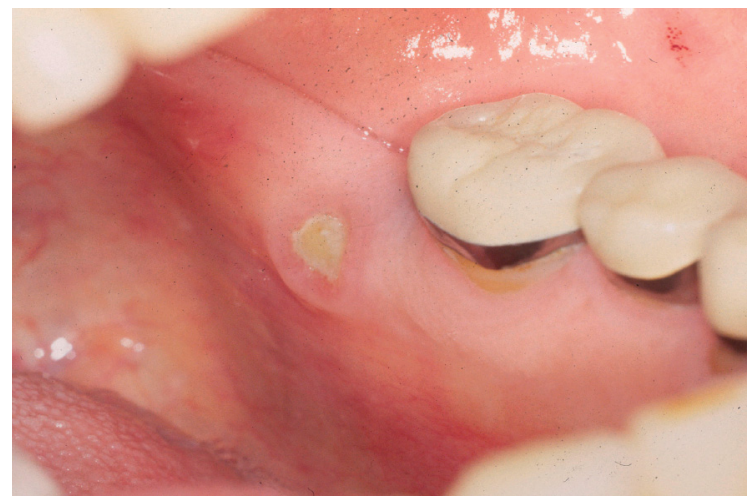

Figure 13: Exposition osseuse au-dessus de la ligne mylo-hyoïdienne, distale à la 36 (cas n ${ }^{\circ}$ 9).

Bone exposure below the mylohyoid line, distal to the first lower left molar (case $n^{\circ}$ 9). médecine

buccale

chirurgie

buccale

VOL. $15, N^{\circ} 1$ 2009

page 55 


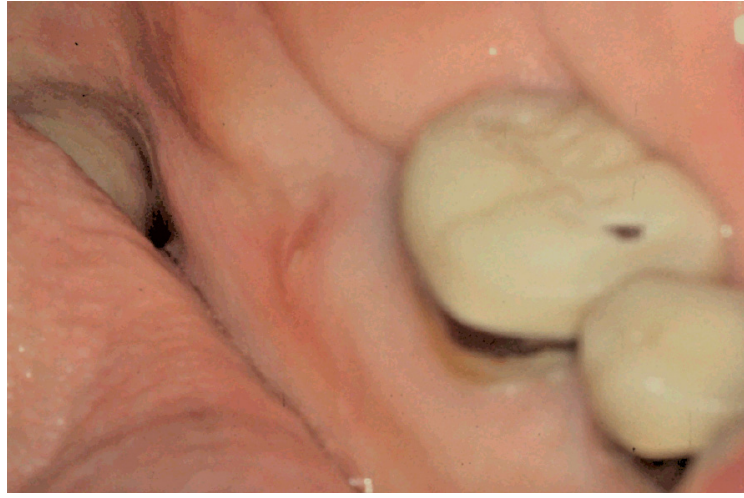

Figure 14 : Aspect de la zone d'exposition, 19 mois après l'élimination du séquestre : persistance d'une légère dépression (cas $n^{\circ} 9$ ).

The exposure area 19 months after removing of the bone sequestrum : persistence of a concavity following bone healing (case $n^{\circ} 9$ ).

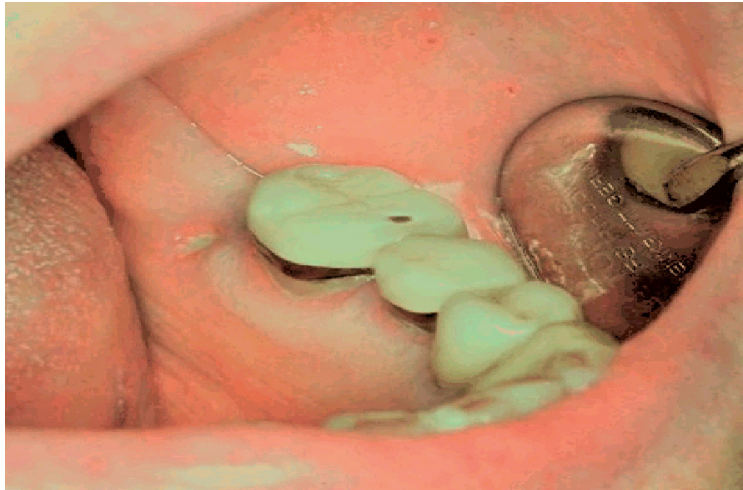

Figure 16: Nouvelle exposition osseuse dans la même région, 31 mois plus tard, mais plus proche du sommet de la crête alvéolaire (cas $n^{\circ}$ 9).

New bone exposure in the same area, but nearer to the top of the alveolar crest, 31 months later (case $n^{\circ}$ 9). médecine

buccale

chirurgie

buccale

VOL. $15, \mathrm{~N}^{\circ} 1$ 2009

page 56

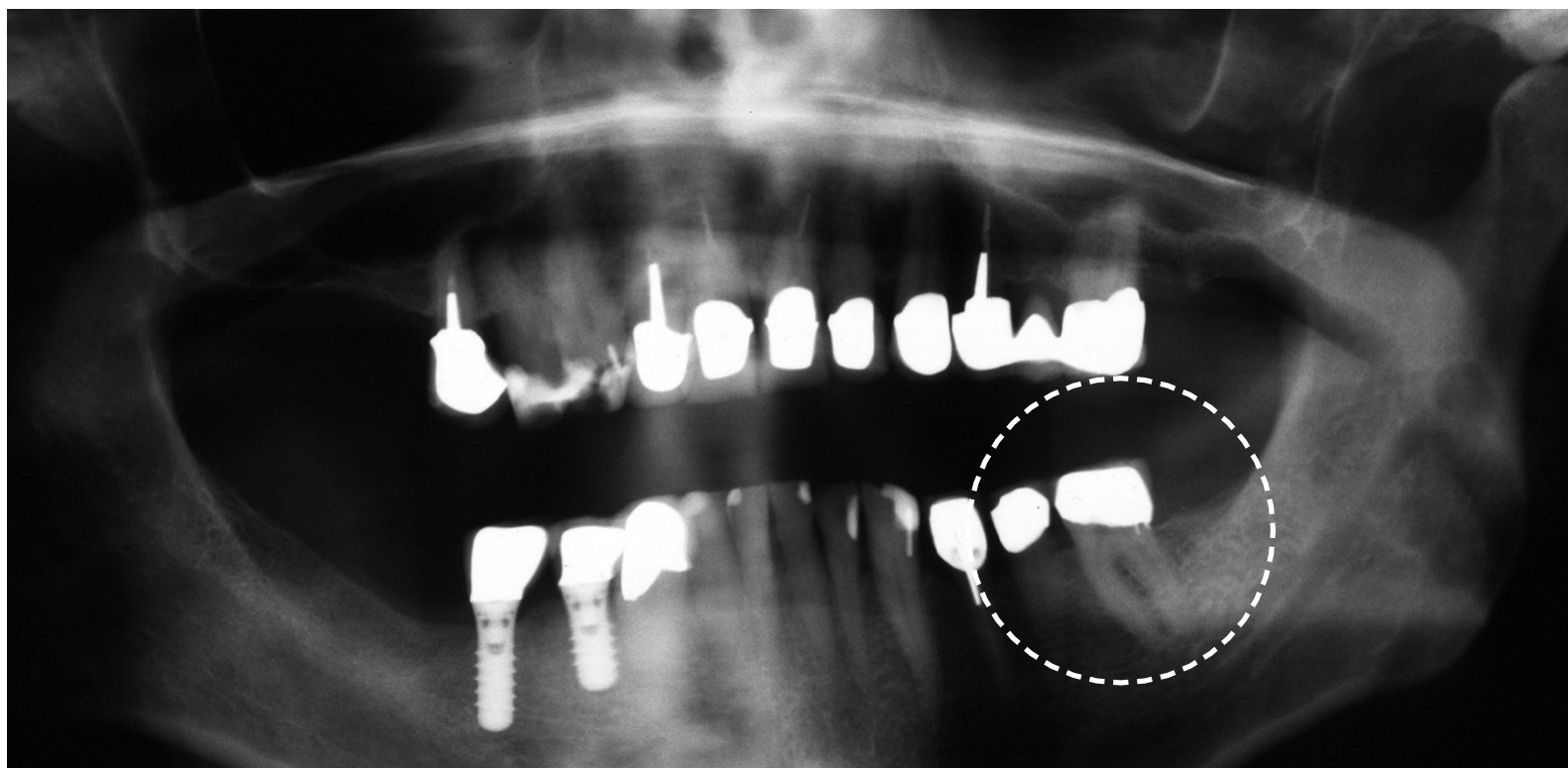

Figure 15: Sur l'orthopantomogramme, la structure osseuse apparait normale dans la zone d'exposition osseuse (cas $n^{\circ}$ 9).

Normal bone structure at the area of the previous bone exposure on the orthopantomogram (case $n^{\circ} 9$ ).

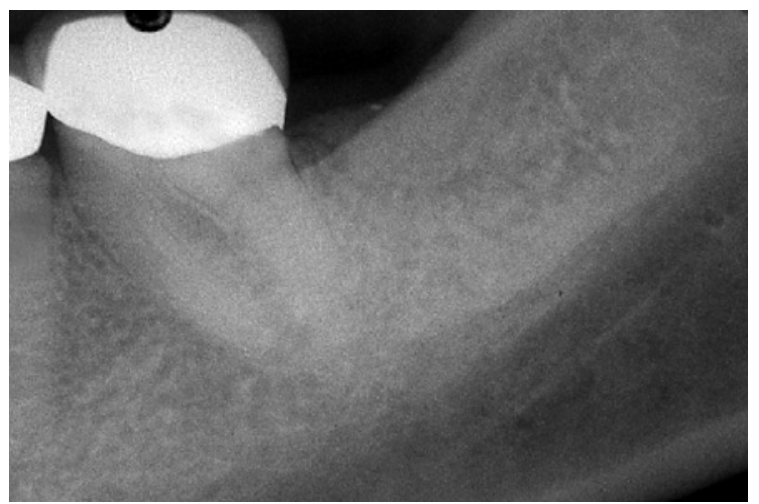

Figure 17 : Absence d'anomalie de la structure osseuse sur la radiographie apicale (cas $n^{\circ}$ 9). Absence of radiological signs of pathology of the bone structure on the periapical radiograph (case $n^{\circ} 9$ ). 
comme la tête du fémur. L'inflammation qui accompagne ou induit le processus de nécrose, se traduit par l'apparition d'un érythème, d'un œdème et de vives douleurs qui précèdent le développement d'une ulcération permettant l'élimination transépithéliale de l'os nécrosé.

L'évolution spontanée aboutit à la guérison (cicatrisation muqueuse) après élimination du séquestre.

\section{RÉFÉRENCES}

1 - Farah CS, Savage NW. Oral ulceration with bone sequestration. Aust Dent J 2003 ; 48 : 61-4.

2 - Flaitz CM. Oral and maxillofacial pathology case of the month. Lingual mandibular sequestration and ulceration. Dent J $2000 ; 117: 40-1$.

3 - Gaudy JF. Anatomie clinique, $2^{e}$ ed. CdP Wolters Kluwer, Rueil-Malmaison, 2007.

4 - Gray H, Warren HL. Anatomy of the human body, 20th ed. Lea and Febiger Philadelphia, New York, 2000.

5 - Jackson I, Malden N. Lingual mucosal ulceration with mandibular sequestration. Dent Update 2007 ; 34 : 5734, 576-7.

6 - Marx RE, Sawatari Y, Fortin M, Broumand V. Bisphosphonate-induced exposed bone (osteonecrosis/osteopetrosis) of the jaws: risk factors, recognition, prevention, and treatment. J Oral Maxillofac Surg 2005 ; $63: 1567-75$.
Une évolution plus rapide peut être obtenue par une prise en charge adéquate associant traitement antalgique, antibiothérapie et exérèse chirurgicale de l'os nécrosé. Une corticothérapie per os précoce, c'est-à-dire dès l'apparition des douleurs, semble pouvoir stopper le processus pathologique et éviter l'évolution vers l'exposition osseuse et la formation d'un séquestre.

7 - Peters E, Lovas GL, Wysocki GP. Lingual mandibular sequestration and ulceration. Oral Surg Oral Med Oral Pathol $1993 ; 75$ : 739-43.

8 - Scully C. Oral ulceration: a new and unusual complication. Br Dent J 2002 ; 192 : 139-40.

9 - Sonnier KE, Horning GM. Spontaneous bony exposure: a report of 4 cases of idiopathic exposure and sequestration of alveolar bone. J Periodontol 1997 ; 68 : 75862

10 - Malizos KN, Karantanas AH, Varitimidis SE, Dailiana ZH, Bargiotas K, Maris T. Osteonecrosis of the femoral head: etiology, imaging and treatment. Eur J Radiol 2007 ; $63: 16-28$. médecine buccale chirurgie buccale

VOL. $15, N^{\circ} 1$ 2009

page 57 